\title{
Analysis of Network Effect in Port and Shipping System Characterized by Scale-free Network
}

\author{
Tian Wei, Guishi Deng, Peijian Wu \\ System Engineering Institute, Dalian University of Technology, Dalian 116023, P. R. China
}

\begin{abstract}
Based on the brief summarization of the development, properties and representative research achievements of scale-free networks, the paper makes an empirical analysis in some complex network characteristics of global shipping lines network. Taking port as dominant products, and shipping service, its complementary products, the mechanism of network effect in products system is analyzedby the study of two-stage budgeting procedure. Then, the paper presents that some complex network characters in a certain domain come from the influence of network benefits in the products network.
\end{abstract}

Keywords: Complex network, Network effect, Twostage budgeting procedure, Shipping network

\section{Introduction}

A wide range of systems in nature and society can be described as complex networks. Recently, complex network has become a hot issue for the research of complex system's topology structure and behavior. In the background of small-world network [1] and scalefree network [2], the research of complex network has made remarkable progress. Foreign researchers have presented some models and conclusions in various fields and industries, including Newman, Moore, May and Lloyd' researches on the spread of disease and virus in small-world network and the infection dynamics on scale-free networks [3]-[6]. In addition, R. Guimer has analyzed the world-wide air transportation network of U.S [7], and Sen P's has also achieved the small-world properties of the Indian railway network [8]. Domestic scholars have also made some research on the theories of the complex system, such as Zhou Tao's research on the spread of SARS and Di Zeng-ru's research on the public transportation network [9]-[11].

Up to the present, the research on the topological structure, grow mechanism and dynamics of network have not set up a perfect theoretical frame. Therefore, most researches have focused on the statistics characteristics analysis on mathematics and physics and these researches depend on the simulation of some statistic phenomenon. Namely, the researchers have continuously improved the models to accurately fit realistic circumstances. However, the researches on the essence of complex network's formation are not available. However, we consider that the statistic properties of complex network are due to different endogenous mechanism in different fields. Some networks which have some properties of complex network may not necessarily belong to the category of typical complex network mentioned above. If we focus our research on the network in a given field, we may come to more comprehensive conclusions. Therefore, we explore the reasons of the statistic properties of small-world and scale-free by combining the complex network theory to a certain network and we hope to provide some realistic foundations for the theory.

\section{The analysis of properties of world-wide shipping network}

Many decisions in the Port industry depend on the formation and development of shipping lines. Whether a port becomes an important also for of some main shipping lines or not is a crucial factor in the programming of the port. How to properly program the port and the shipping industry in the keen competition has become an urgent, world-wide problem.

\subsection{The world-wide shipping network topology}

The Statistics of Brs-Alphaline, a senior ship broker corporation in France, show that the container cargo of the top 25 liner shipping Companies covers the $81.3 \%$ of the total amount in 2004. Thus, we assume that the total lines statistics of these 25 Liner Shipping Company can reflect the current situation of international shipping. Our statistics are, therefore, composed of 1400 lines and more 500 also for 
provided by the top 25 companies in their official websites.

Taking also for in main lines as node, and shipping lines the edge, the topology structure of world-wide shipping network can be described as the Fig.1.

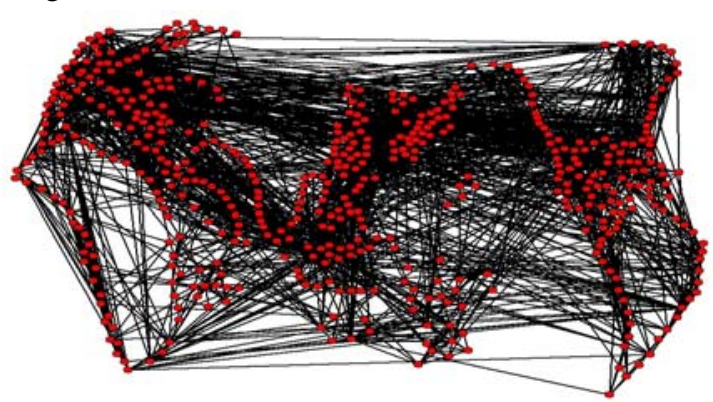

Fig.1: Topology of the world-wide shipping network.

Owing to the radiate area of the port, the distribution of natural resources and the economic conditions of the surrounding area of the port, the port with larger connection numbers is usually located in dispersive areas. The ports and the lines present a network structure with multi-community. The large connection numbers nodes are the hub of the community. The hub port's operating condition will influence the other ports in the same community

\subsection{The properties of small-world and scale-free in world-wide shipping network}

The small world concept in simple terms describes the fact that in most networks despite the large number of nodes they are made of, the typical distance between two nodes is very short. The distance between two nodes in a network is defined as the number of edges in the shortest path connecting them. The most popular manifestation of "small worlds" is the "six degrees of separation" concept, uncovered by the social psychologist Stanley Milgram (1957).

As Fig. 2 shows, we find the distribution of shortest path between ports and get the average path length $l=3.252$ in world-wide shipping network.

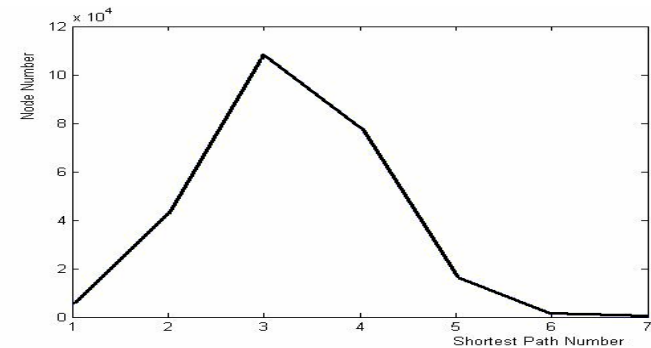

Fig.2: Distribution of shortest path between ports.
The Fig.2 also shows us that the maximum path length between any two ports is 7, and almost any two ports in the world-wide shipping network can be connected within 6 steps. We can thus conclude that the world-wide shipping network has typical property of small-world.

After much calculation, we get the clustering coefficient $C=0.4$ in the shipping network, and the clustering coefficient of the network is presented in Fig.3.

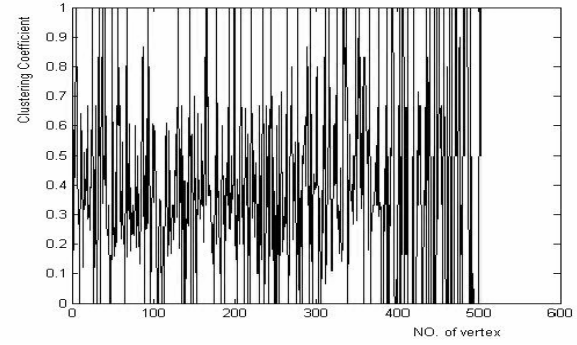

Fig.3: Clustering coefficient of ports in the shipping network.

Based on the analysis of the world-wide shipping network, we can conclude that the network shows negative clustering-degree relationship (correlation coefficient $r=-0.17$ ) and positive degree-degree relationship ( $r=1.15$ ). Negative clustering-degree relationship shows that the hub ports in the shipping network plays an important role. Although many ports connect with the hub ports, they have no close relation among themselves. The positive degree-degree relationship shows that the ports with higher degree are more likely to connect with each other.

Some ports' clustering coefficient is smaller than others due to the economy of programming and operation of shipping lines. If two ports are close to each other, and a certain main line has already connected these two ports, Liner Shipping Company will not set another new line to connect them in order to maximize the payoff and minimize the shipping cost .

Fig.4 shows the degree distribution of the ports in the network.

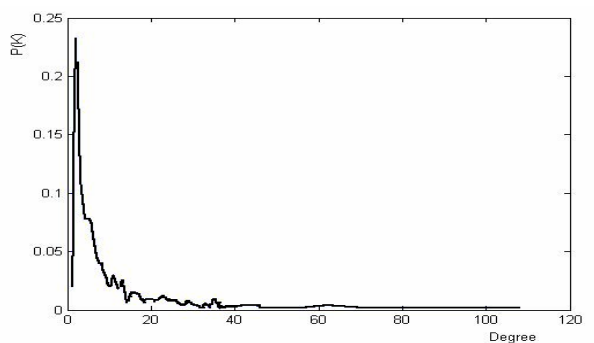

Fig.4: Degree distribution of ports in the shipping network.

According to Fig.4, we find that the distribution of the world-wide shipping network's degree is similar 
to power-law property. Furthermore, Fig. 5 presents the result of curve approximation to the distribution of degree. The probability that a node has a degree $k$ can be written as $p(k)=0.2873 k^{-1.38}$. The equation indicates that the degree of all ports evolves the same way, following a power-law.

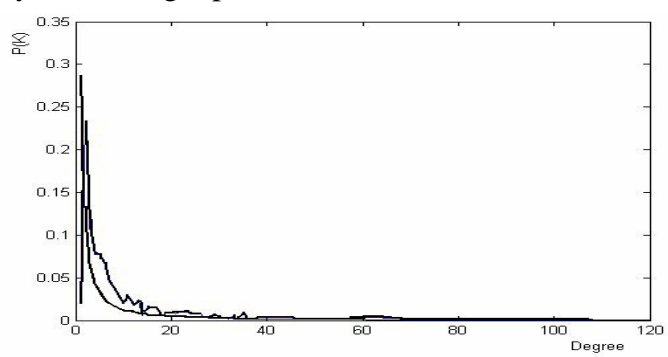

Fig.5: Power-law prosperity of the degree distribution.

\subsection{Analysis of the properties similar to the complex network}

From the above analysis of the world-wide shipping network, we can find that the shipping network has some properties of complex network, such as short average path length and power-law of degree distribution. Since the clustering coefficient of the network is not very large and the clustering coefficient of some ports is too small, the network is not completely consistent with the typical complex network. However, the difference shows the exact conditions of the shipping network. The phenomenon shows that politics, geography, economy and so on exert great influence on the programming of the shipping lines, which works against the formation and the operation mechanism of the shipping network.

However, we consider that network's simulation and modeling fail to explain the intrinsic reason accounting for these properties similar to the complex network. Recently, many scholars have built some generated models with quite a few restrictions so as to accurately reflect the history and currently conditions of the network. In fact, we believe that these kinds of models are just simulations of the reality, but not the analysis of the essential reasons for the small-world and scale-free properties.

Unlike the methods mentioned above, we present a new idea to explore why and how the small-world and scale-free properties generate. Based on the analysis of world-wide shipping network and the conditions of the port industry, we take port as the dominant product and services of port and shipping as its complementary products in the product network.

Once a shipping line company chooses a port (dominant product) as its main also for, the port tend to connect with more ports. At the same time, the port and shipping services (complementary products) will get more chance to increase its profits.

We try to analyze the factors influencing the product's utility, and ultimately map the utility analysis to the ports and shipping network so as to provide some decision support information for shipping programming and management.

\section{The utility analysis of the network products}

\subsection{The definition and classification of network effect}

Rohlfs, the economist, found the typical case of the consuming external economy in communication industry in 1974, and presented the cumulate demand function of communication service under the influence of external economy conditional on the same preference of users [12]. The research established the theoretical foundation of the network effect.

Based on the different methods of feedback, Katz and Shapiro categorized the network effect into direct and indirect network effects [13][14]. In the presence of direct network effect, the increase in the number of users (the network size) of a product raises the value of the product directly. For example, in telecommunication and software industries, the consumer's utility level depends directly on the size of the network. On the other hand, indirect network effects are caused by the consumer's preference for the variety and quality of complementary products available for a dominant product.

\subsection{The definition of network benefit function}

Not only Katz and Shapiro but also Farrell and Saloner simultaneously presented the utility function of network products in 1985 [15]. The function can be described as $U=r+v\left(x^{e}\right)$ to denote the utility of consumer's preference. Where $r$ is the invariable elementary utility of the products, and it is not relevant to the installed base of network. Where $v\left(x^{e}\right)$ is called the network benefit function to denote the network benefit for consumers brought by the anticipated network size $x^{e}$.

In the presence of direct network effect, network benefit is linear in the network size, and the network benefit is concave and monotonous. The previous studies of indirect network externalities have usually postulated the consumer's network benefit as an 
increasing (and concave) function of the variety of available complementary products (e.g., Chou and Shy, 1996, etc.)[16][17].This direct specification of the consumer's benefit has been successfully utilized in the study of the dominant producer's control over complementary products provisions. However, this specification cannot fully reflect positive feedback effects of indirect network effects in which increased sales of a dominant product are re-enforced by independent complementary products producers' provision of a greater variety of available complementary products.

Therefore, we denote the indirect network effects as the function of the independent income $V_{i j}$, coefficient $\lambda$, anticipated network size $E\left[B_{j}\right]$ through Dixit and Stiglitz's two-sector utility function [18].

\subsection{Analysis of network benefit function of indirect network effects}

In what follows, let $V_{i j}$ denote consumer $i$ 's standalone benefit of dominant product $j ; \mathrm{Z}_{\mathrm{ik}[\mathrm{j}]}$ denote the quantity consumed by consumer $\mathrm{i}$ of complementary product $k$ available for dominant product $\mathrm{j}$. $K_{j}$ denote the variety (or the number) of complementary products available for dominant product $\mathrm{j}$; and $q_{i o}$ denote the quantity consumed by consumer $\mathrm{i}$ of the outside alternative.

For notational simplicity, we let $Z_{i k}$ abbreviate $\mathrm{Z}_{\mathrm{ik}[\mathrm{j}]}$.

Define $\mathrm{Z}_{i j}=\left(\sum_{i=1}^{k_{j}} Z_{i k}^{1 / \beta}\right)^{\beta}$ with $\beta>1$.

And we assumed the utility level of consumer $i$ for products system $j$ is given by the scalar value: $U_{i j}=V_{i j}+f\left(Z_{i j}\right)+q_{i o}$ where $f$ is a real function and $g \equiv f^{\prime}>0, \quad g^{\prime} \equiv f^{\prime \prime}<0$.

The utility function for various available complementary products and the outside alternative, $f\left(Z_{i j}\right)+q_{i o}$, can be considered as a special form of the two-sector utility function in Dixit and Stiglitz. " $\beta>1$ " guarantees concavity of the utility function in the number of complementary products, and is sufficient to ensure that the marginal rate of substitution between $q_{i o}$ and $Z_{i j}$ is decreasing.

In order to reflect indirect network effects as a positive consumption externality, we impose a restriction on the functional form of $f$ as $-\frac{\partial g^{-1}}{\partial Y}<\frac{\beta}{\beta-1} \frac{Z_{i j}}{g\left(Z_{i j}\right)}$, where $Y=g\left(Z_{i j}\right)$. We can conclude that with these two assumptions ensure the consumer prefers a greater variety of available complementary products, and more users of a dominant product induce a greater variety of available complementary products.

Let $B_{j}$ denote the network size of dominant product $j$ in the beginning of the second stage. In the second stage, consumer $i$ who purchased dominant product $j$ splits the remainder of his income between available complementary products, $\left\{Z_{i k}\right\}$, and the outside alternative, $q_{i o}$, to get:

$$
\max : V_{i j}+f\left(Z_{i j}\right)+q_{i o} \text {. }
$$

Here, the restriction of the maximum is

$$
q_{i o}+\sum_{K=1}^{K_{j}} \rho_{k} Z_{i k}=y_{i}-p_{j}
$$

where $\rho_{k}$ denote the price of complementary product $k, y_{i}$ is consumer $i$ 's income, and $p_{j}$ denotes the price of dominant product $j$.

Following a two-stage budgeting procedure we derive a consumer's demand function for an available complementary product as follows:

$$
Z_{i k}=g^{-1}\left(P_{j}\left[\frac{P_{j}}{\rho_{k}}\right]^{\beta /(\beta-1)}\right.
$$

where $P_{j}=\left(\sum_{K=1}^{K_{j}} \rho_{k}^{-1 /(\beta-1)}\right)^{-(\beta-1)}$.

Since $g$ is a decreasing function, $g^{-1}$ exists and is also a decreasing function. A consumer's demand function for an available complementary product $Z_{i k}$ does not depend on the consumer's income level since the utility function is specified as linear in the outside alternative. And we the present price elasticity of demand for complementary product $k$ :

$$
-\left(\partial Z_{i k} / \partial \rho_{k}\right)\left(Z_{i k} / \rho_{k}\right)=\beta /(\beta-1) \text {. }
$$

Each complementary maker is assumed to produce one complementary product with the same marginal cost $s$, and the same fixed cost $F$. Then the first order condition of the complementary producer's profit maximization leads to:

$$
\rho_{k}=\beta s=\rho
$$

Substituting the prices of complementary products in (3) into the consumer's demand function in (2) yields:

$$
Z_{i k}=g^{-1}\left(\rho K_{j}^{-(\beta-1)}\right) K_{j}^{-\beta}
$$

Substituting the consumer's demand functions for complementary products in (4) into the budget 
constraint in (1), we derive a consumer's demand function for the outside alternative as follows:

$$
q_{i o}=y_{i}-p_{j}-\rho g^{-1}\left(\rho K_{j}^{-(\beta-1)}\right) K_{j}^{-(\beta-1)}
$$

A variety of available complementary products, $K_{j}$, is endogenously determined by the zeroprofit condition imposed by free entry.

Substituting both the consumer's demand functions for available complementary products in (4) and the consumer's demand function for the outside alternative in (5) into the utility function in (1), we derive the indirect utility function for products system as follows:

$$
U_{i j}=v_{i j}+y_{i}-p_{j}+\lambda\left(B_{j}\right)
$$

where network benefit function

$$
\lambda\left(B_{j}\right)=-\rho g^{-1}\left(\rho K_{j}^{-(\beta-1)}\right) K_{j}^{-(\beta-1)}+f\left(g^{-1}\left(\rho K_{j}^{-(\beta-1)}\right)\right)
$$

The coefficient $\lambda$ of the network benefit function calculated as follow.

$$
\begin{aligned}
& \text { Suppose that } f\left(Z_{i j}\right)=Z_{i j}^{1 /(2 \beta-1)} \text {, then } \\
& Y \equiv g\left(Z_{i j}\right) \equiv f^{\prime}\left(Z_{i j}\right)=(2 \beta-1)^{-1} Z_{i j}^{-2(\beta-1) /(2 \beta-1)},
\end{aligned}
$$

and

$$
g^{-1}(Y)=Z_{i j}=[(2 \beta-1) Y]^{-(2 \beta-1) /(2 \beta-2)} .
$$

Therefore,

$$
\frac{\partial g^{-1}}{\partial Y}=-\frac{1}{2(\beta-1)}(2 \beta-1) \frac{-1}{2 \beta-1} g\left(Z_{i j}\right) \frac{4 \beta-3}{2(\beta-1)}
$$

From the condition s of (7), (8) and $\beta>1$ we know the assumption of $f\left(Z_{i j}\right)$ is satisfied above assumption of $-\frac{\partial g^{-1}}{\partial Y}<\frac{\beta}{\beta-1} \frac{Z_{i j}}{g\left(Z_{i j}\right)}, Y=g\left(Z_{i j}\right)$

Substituting both the (7) and the zero-profit condition and the consumer's demand function in (4) into network benefit function $\lambda\left(B_{j}\right)$, we derive the coefficient $\lambda$ can be rewritten as follows:

$$
\lambda=\frac{\beta-1}{F}(2+s)(\beta s)^{-\frac{\beta}{\beta-1}}(2 \beta-1)^{-\frac{2 \beta-1}{\beta-1}}
$$

The conclusion shows that coefficient $\lambda$ of network benefit function depends on the marginal cost, fixed cost and the coefficient $\beta$ (consumers' variety preference) of consumers' utility function.

In conclusion, the intensity of the indirect network effects can be described as linear utility function similar to the direct network effects. However, the indirect network effects depend on the cost and consumers' variety preference rather than the installed base.

\section{Conclusions}

Being restricted by the scale of the data and the simplifying process of the weight of the data, we make an empirical analysis to get the conclusion that the shipping network has some properties similar to complex network. However, we can not prove that the world-wide shipping network belongs to the typical category of complex network. In addition, we believe that statistic methods and modeling cannot accurately define the formation mechanism of the shipping network as well as other similar networks. Moreover, we cannot predict the development of this kind of network with help of the above methods.

Based on the above utility analysis of network effects, taking the port as the network product system (shipping and relevant port service system), the shipping companies, the consumers of products, we consider that the statistic properties such as the scalefree property in certain fields (society, economy and communications) come from consumers' pursuit for the utility. The utility comes from installed base of the product network, the cost of the product system and the variety of consumers' preference. In other word, the statistic properties of the shipping network are due to the influence exerted by the network benefit of the product system on the utility of the products.

Therefore, in a port industries district with external economic effects, the port should draw the resources, the economic factors in the surrounding area so as to provide differentiated products through various operating methods. In order to improve the utility and the competitive advantage of the port, we should try best to reduce the production cost of the port and improve the quality of the port and shipping services.

\section{Acknowledgement}

This work is partially supported by National Nature Science Foundation of China (Grant No. 70671016).

\section{References}

[1] D.J. Watts and S.H. Strogatz, Collective dynamics of small world networks. Nature, 393:440-442, 1998.

[2] R. Albert and A.L. Barabási, Statistical mechanics of complex networks. Review of Modern Physics, 74:47-97, 2002.

[3] M.E.J. Newman, The structure and function of complex networks. SIAM Review, 45:167-256, 2003.

[4] M.E.J. Newman, Spread of epidemic disease on networks. Phys Rev E, 66:016128, 2002. 
[5] C. Moore and M.E.J. Newman, Epidemics and percolation in small-world networks. Phys Rev E, 61:5678-5682, 2000.

[6] R.M. May and A.L. Lloyd, Infection dynamics on scale-free networks. Phys Rev E, 64:066-112, 2001.

[7] R. Guimera and S. Mossa, A. Turtschi and L.A.N. Amaral, The world-wide air transportation network: Anomalous centrality, community structure, and cities' global roles. Proc. Nat. Acad. Sci. USA, 102:7794-7799, 2005.

[8] P. Sen, P. Dasgupta, A. Chatterjee, et al, Smallworld properties of the Indian railway network. Phys Rev E, 67: 036106, 2003.

[9] G. Yan, T. Zhou, J. Wang , et al, Epidemic spread in weighted scale-free networks. Chinese Physical Letter, 22:510-513, 2005.

[10] T. Zhou, An overview of dynamics on complex network. Progress in Natural Science, 15:513518, 2005.

[11] J.S. Zhao, Z.R. Di, D.H. Wang, Empirical research on public transport network of Beijing. Complex Systems and Complexity Science, 2:4548, 2005.

[12] J.A. Rohlfs, , Theory of interdependent demand for a communications service. Bell Journal of Economics, 5:16-37, 1974.

[13] M.L. Katz and C. Shapiro, Product introduction with network externalities. Journal of Economic Perspectives, 40:55-83, 1992.

[14] M.L. Katz and C. Shapiro, Network externalities, competition, and compatibility. American Economic Review, 75:424- 440, 1985.

[15] J. Farrell, G. Saloner, Standardization, compatibility, and innovation. Rand journal of Economic, 16:70-83, 1985.

[16] C. Chou and O. Shy, Do consumers gain or lose when more people buy the same brand? European Journal of Political Economy, 12:309330, 1996.

[17] J. Church and N. Gandal, Network effect, software provision and standardization. Journal of Industrial Economics, 40:85-104, 1992.

[18] A. Dixit and J. Stiglitz, Monopolistic competition and optimal product diversity. American Economic Review, 67:297- 308, 1977.

[19] N. Economides, Minimal and maximal product differentiation in Hotelling's duopoly. Economic Letters, 21:67- 71, 1986.

[20] J.M. Yang, S.J. Wang and L.P. Lu, Study of social network and complex competitive relationship network of software industry in Guangzhou. Chinese Journal of Management, 6:723-727, 2006.
[21] W. Li and X. Cai, Statistical analysis of airport network of China. Phys Rev E, 69:046106, 2004. 